\title{
Validação do Questionário Otitis Media-6 para Português Europeu
}

\section{Validation of the Otitis Media-6 Questionnaire for European Portuguese}

\author{
Ana Rita LAMEIRAS $\triangle^{1}$, Deodato SILVA ${ }^{1}$, Assunção O'NEILL ${ }^{1,2,3}$, Pedro ESCADA ${ }^{1,3}$ \\ Acta Med Port 2017 May;30(5):381-387 - https://doi.org/10.20344/amp.7921
}

\section{RESUMO}

Introdução: A otite média é uma das doenças mais prevalentes na infância. O impacto da otite média na qualidade de vida das crianças portuguesas não é conhecido, devido à indisponibilidade de um instrumento validado em português europeu capaz de avaliar esta consequência da otite média. O questionário Otitis Media-6 é o instrumento mais frequentemente usado para avaliar a qualidade de vida relacionada com a saúde em crianças com otite média. Este estudo tem como objetivo criar uma versão em língua portuguesa $e$ culturalmente adaptada a Portugal do questionário Otitis Media-6.

Material e Métodos: O questionário Otitis Media-6 foi traduzido e culturalmente adaptado à língua e à população portuguesa. Em seguida, para avaliar as propriedades psicométricas do instrumento, o mesmo foi aplicado a uma população de crianças portuguesas com otite média crónica com efusão ou otite média aguda recorrente.

Resultados: A versão portuguesa do questionário Otitis Media-6 demonstrou as seguintes propriedades psicométricas: validade de construção para os scores basal $\left(r_{s}=0,98\right)$ e de mudança $\left(r_{s}=0,97\right)$, consistência interna $(\alpha=0,780)$, confiança teste-reteste $\left(r_{s}=0,89\right)$ e resposta a alteração clínica $(t(59)=10,104)$.

Discussão: A simplicidade e brevidade de aplicação do instrumento tornam-no ideal para utilização em investigação e na prática clínica diária, possibilitando uma avaliação mais objetiva da extensão do impacto da otite média na qualidade de vida da criança e a tomada de decisões terapêuticas mais orientada.

Conclusão: A versão portuguesa do questionário Otitis Media-6 é um instrumento válido, confiável e sensível para avaliação da qualidade de vida relacionada com a saúde em crianças portuguesas com otite média.

Palavras-chave: Criança; Inquéritos e Questionários; Otite Média; Portugal; Qualidade de Vida

\section{ABSTRACT}

Introduction: Otitis media is one of the most prevalent childhood diseases. The impact of otitis media on quality of life of Portuguese children is unknown, because of the unavailability of a tool validated in European Portuguese to assess this consequence of otitis media. The Otitis Media-6 questionnaire (Otitis Media-6) is the most frequently used tool to assess health-related quality of life in children with otitis media. This study aims to create a version in the Portuguese language and culturally adapted to Portugal of the otitis media-6 questionnaire.

Material and Methods: The Otitis Media- 6 questionnaire was translated and culturally adapted to the Portuguese language and population. Then, to assess the instrument psychometric properties, it was applied to a sample of Portuguese children with chronic otitis media with effusion or recurrent acute otitis media.

Results: The Portuguese version of Otitis Media-6 questionnaire demonstrated the following psychometric properties: construct validity for baseline $\left(r_{s}=0.98\right)$ and change scores $\left(r_{s}=0.97\right)$, internal consistency $(\alpha=0.780)$, test-retest reliability $\left(r_{s}=0.89\right)$ and responsiveness to clinical change $(t(59)=10.104)$.

Discussion: The simplicity and brevity of application of the instrument make it ideal for use in research and in clinical practice, enabling a more objective assessment of the extension of the otitis media impact in children quality of life and a more targeted therapeutic decision.

Conclusion: The Portuguese version of the Otitis Media- 6 questionnaire is a valid, reliable and sensitive instrument to evaluate the health-related quality of life in Portuguese children with otitis media.

Keywords: Child; Otitis Media; Portugal; Quality of Life; Surveys and Questionnaires

\section{INTRODUÇÃO}

A otite média (OM) é uma doença comum da infância e uma das principais causas de observação médica em crianças em idade pré-escolar. ${ }^{1}$ As duas principais formas de OM em idade pediátrica são a otite média crónica com efusão e a otite média aguda, observando-se frequentemente um contínuo entre estas duas entidades clínicas. ${ }^{2}$

A maioria dos estudos que avaliam o impacto da OM, baseiam-se em medidas fisiológicas, tais como o limiar auditivo, a taxa de recorrência de OM, o estado do ouvido médio e a integridade da membrana timpânica. Contudo, a importância da consideração da qualidade de vida tem sido reconhecida de forma crescente na investigação clínica sobre OM. ${ }^{3}$ De facto, os episódios repetidos de otalgia, febre e mal-estar geral ${ }^{4,5}$ ou as possíveis sequelas a longo-prazo, tais como perda auditiva e alteração do desenvolvimento da linguagem, 6,7 podem afetar a qualidade de vida relacionada com a saúde em crianças com OM.

O questionário Otitis Media-6 (OM-6), originalmente

1. Serviço de Otorrinolaringologia. Hospital de Egas Moniz. Centro Hospitalar de Lisboa Ocidental. Lisboa. Portugal.

2. Departamento de Anatomia. NOVA Medical School. Universidade Nova de Lisboa. Lisboa. Portugal.

3. Departamento de Otorrinolaringologia. NOVA Medical School. Universidade Nova de Lisboa. Lisboa. Portugal.

$\square$ Autor correspondente: Ana Rita Lameiras. rita_lameiras@hotmail.com

Recebido: 08 de junho de 2016 - Aceite: 06 de fevereiro de 2017 | Copyright @ Ordem dos Médicos 2017 
desenvolvido por Rosenfeld et aß em língua inglesa, é o instrumento mais usado para avaliação da qualidade de vida relacionada com a saúde em crianças com OM, encontrando-se já traduzido e validado para outras línguas. ${ }^{8-10}$ Este trabalho tem como objetivo a criação de uma versão em língua portuguesa e culturalmente adaptada a Portugal do questionário OM-6 e a avaliação da sua consistência e fiabilidade.

\section{MATERIAL E MÉTODOS}

Este trabalho é um estudo longitudinal prospetivo, realizado no serviço de Otorrinolaringologia do Hospital de Egas Moniz, Centro Hospitalar de Lisboa Ocidental. Foi desenvolvido em duas fases: Fase I - tradução e adaptação cultural do questionário OM-6; Fase II - aplicação da versão portuguesa (português europeu) do questionário OM-6 a crianças portuguesas com OM com o objetivo de avaliar a sua consistência e fiabilidade. Foi obtido consentimento escrito para tradução e aplicação do questionário junto do primeiro autor da versão original do questionário OM-6. ${ }^{3}$

O presente trabalho foi autorizado pela Comissão de Ética da instituição onde o mesmo foi realizado, de acordo com a Declaração de Helsínquia.

\section{Caracterização do questionário}

O questionário OM-6 tem como população-alvo crianças com idade compreendida entre seis meses e 12 anos, com otite média crónica com efusão (com duração $\geq$ três meses) ou otite média aguda recorrente ( $\geq$ três episódios nos últimos 12 meses, com normalização completa da otoscopia nas intercrises). O questionário OM-6 é preenchido pelo cuidador e avalia seis domínios relativos à OM: sofrimento físico, perda auditiva, comprometimento da fala, sofrimento emocional, limitação da atividade e preocupação do cuidador. Cada domínio é representado por um único item com sete opções de resposta que permitem avaliar as diferentes formas de afeção da qualidade de vida pela OM nas últimas quatro semanas. As respostas aos seis itens são pontuadas de 1 a 7 (1 - sem problema; 7 - problema extremo). A pontuação de cada item é somada e dividida por seis para obter uma pontuação final (entre 1,0 e 7,0). Quanto mais elevada a pontuação final, pior a qualidade de vida relacionada com OM.

Pode ser calculado o score de mudança, através da subtração da pontuação final obtida no questionário após intervenção (score de follow-up) - por exemplo, tratamento cirúrgico com miringotomia e colocação de tubos de ventilação transtimpânicos, à pontuação final obtida antes da intervenção (score basal) (score de mudança = score basal - score de follow-up). O score de mudança permite avaliar a resposta a determinada terapêutica, com um intervalo mínimo entre as avaliações de quatro semanas. Um score de mudança negativo indica um agravamento clínico e um score de mudança positivo indica uma melhoria clínica. Encontram-se definidos intervalos de score de mudança para avaliar o grau de alteração com uma intervenção específica: < 0,5 - trivial; 0,5 - 0,9 - pequena; 1,0 - 1,4 - moderada; $\geq 1,5$ - grande.

\section{Fase I}

A versão original do questionário OM-6, em língua inglesa, foi objeto de avaliação por dois médicos da especialidade de Otorrinolaringologia, bilingues (português e inglês) de língua nativa portuguesa (português europeu) e utilizadores independentes da língua inglesa (nível B2), tendo sido produzida uma primeira versão portuguesa do questionário OM-6. Esta versão foi alvo de apreciação por parte de um painel, composto pelos dois tradutores e por outros três médicos da especialidade de Otorrinolaringologia, que levou à adaptação de três expressões que se consideraram inadaptadas à realidade portuguesa. A expressão 'fluid' (consensualmente traduzida como 'fluido') foi transformada em 'derrame (líquido)'. No item relativo ao sofrimento físico, a expressão 'discharge' (consensualmente traduzida como 'descarga') foi transformada em 'purgação (supuração)'. No item relativo à alteração da fala, a expressão 'poor pronunciation' (consensualmente traduzida como 'pronúncia pobre') foi transformada em 'alteração da pronunciação (alteração da articulação da fala)'.

Esta primeira versão portuguesa adaptada, designada como 'primeira tradução de consenso', foi entregue a dois tradutores profissionais, bilingues (português e inglês) e de língua nativa inglesa, a fim de ser traduzida para inglês. Os tradutores desconheciam os objetivos do trabalho e a versão original do questionário OM-6 e não tiveram qualquer contacto entre si. As duas retro-traduções foram analisadas, face à versão inglesa original pelos dois tradutores de língua nativa portuguesa. Desta análise resultou uma 'segunda tradução de consenso'.

A 'segunda tradução de consenso' foi apresentada a um grupo de cuidadores de crianças com OM. Para esse efeito, procedemos à divisão da 'segunda tradução de consenso' em duas partes: primeira (I), relativa às instruções para resposta ao instrumento; segunda (II), relativa às perguntas nele contidas. A segunda parte foi subdividida em seis secções (II1-6) correspondentes aos seis itens. Para cada uma delas foi solicitada a opinião do cuidador quanto à clareza do texto: 'percebo muito bem', 'percebo bem', 'percebo mal' ou 'percebo muito mal'. Estabelecemos um cut-off para reformulação dos itens da 'segunda tradução de consenso' de $>15 \%$ de respostas 'percebo mal' ou 'percebo muito mal'.

Considerámos os seguintes critérios de inclusão e de exclusão no estudo. Critérios de inclusão: (1) criança com idade compreendida entre seis meses e 12 anos; (2) diagnóstico de otite média crónica com efusão (com duração $\geq$ três meses) ou otite média aguda recorrente ( $\geq$ três episódios nos últimos 12 meses, com normalização completa da otoscopia nas intercrises) uni ou bilateral; (3) cuidador de língua nativa portuguesa (português europeu). Critérios de exclusão: (1) perfuração da membrana timpânica; (2) tubo(s) transtimpânico(s) colocados no início do estudo; (3) outra patologia do ouvido médio (por exemplo, colesteatoma); (4) atraso do desenvolvimento ou patologia 
neurológica; (5) doença sindrómica ou fenda palatina; (6) cuidador incapaz de ler e/ou compreender língua portuguesa (português europeu). Não foram excluídas crianças com antecedentes de colocação de tubos de ventilação transtimpânicos.

O diagnóstico dos diferentes tipos de OM baseou-se na presença dos achados clássicos na otoscopia, observados por médico Otorrinolaringologista habilitado para o efeito, e nos resultados obtidos na timpanometria.

\section{Fase II}

Obtida a versão portuguesa do questionário OM-6, procedemos à sua aplicação a crianças e respetivos cuidadores, de acordo com os critérios de inclusão e exclusão especificados na Fase I, com o objetivo de avaliar a sua consistência e fiabilidade. Durante o estudo, o questionário OM-6 foi completado pelos cuidadores sem assistência.

A validade de construção do instrumento foi avaliada através da comparação da pontuação final obtida no questionário OM-6 com a avaliação global da qualidade de vida relacionada com o estado do ouvido médio numa escala visual analógica de 10 pontos ( 0 - pior qualidade de vida possível, 10 - melhor qualidade de vida possível). Para esse efeito, utilizámos o coeficiente de correlação de Spearman.

A consistência interna foi avaliada pela pesquisa de associações entre os itens do questionário. Com esse objetivo, utilizámos o método alfa de Cronbach, tendo uma boa consistência sido definida por um valor de alfa de Cronbach superior a 0,70 .

A confiança teste-reteste foi avaliada pela aplicação da versão portuguesa do questionário OM-6 no intervalo de sete dias a um subgrupo de crianças com doença estável, definida pela presença de estabilidade clínica (ausência de agravamento ou melhoria do quadro clínico). A distribuição normal das duas pontuações obtidas (pontuação final inicial e pontuação final reteste) foi confirmada através do teste Kolmogorov-Smirnov. Utilizámos o coeficiente de correlação de Pearson para avaliação da confiança teste-reteste, tendo uma boa confiança sido definida por um coeficiente de correlação igual ou superior a 0,70 entre a pontuação final inicial e a pontuação final reteste.

A resposta a alteração clínica foi avaliada numa amostra de doentes com indicação para colocação de tubos de ventilação transtimpânicos como parte do seu seguimento clínico, sendo expectável uma melhoria significativa da qualidade de vida pela eficácia reconhecida do procedimento no tratamento da otite média crónica com efusão e da otite média aguda recorrente. 11,12 $^{11}$ questionário OM-6 e a escala visual analógica de 10 pontos foram aplicados aos respetivos cuidadores antes do procedimento cirúrgico e aos dois meses de pós-operatório. Para analisar a diferença entre as pontuações finais obtidas antes (score basal) e após a colocação de tubos de ventilação transtimpânicos (score de follow-up) no questionário OM-6 utilizámos o teste $t$ de Student para amostras emparelhadas. O score de follow-up de ambos os instrumentos foi completado pelo mesmo cuidador que completou o score basal.

O score de mudança do questionário OM-6 (score basal - score de follow-up) foi calculado nesta amostra de doentes, tendo a validade de construção do mesmo sido avaliada por comparação com o score de mudança da escala visual analógica de 10 pontos (score de follow-up - score basal) através do coeficiente de correlação de Spearman.

Os dados recolhidos foram introduzidos em folha de cálculo Excel ${ }^{\circledR}$, tendo o estudo estatístico sido realizado recorrendo ao Statistical Package for The Social Sciences ${ }^{\circledR}$ (SPSS) versão 21.0 para Windows ${ }^{\circledR}$.

\section{RESULTADOS}

\section{Fase I}

A 'segunda tradução de consenso' foi apresentada a um grupo de 50 cuidadores de crianças com OM. Em nenhum dos itens da 'segunda tradução de consenso' a avaliação, pelo grupo de cuidadores, apresentou > $15 \%$ de respostas 'percebo mal' ou 'percebo muito mal' (Tabela 1), pelo que não houve necessidade de reformulação. Com base nos resultados obtidos foi criada a versão portuguesa do questionário OM-6 (Apêndice 1) [http://www.actamedicaportuguesa.com/revista/index.php/ amp/article/view/7921/5045].

\section{Fase II}

A recolha de dados decorreu durante um período temporal de seis meses. O estudo envolveu uma amostra total de conveniência composta por 216 crianças e respetivos cuidadores. A idade média das crianças variou entre seis meses e 12 anos ( $M=4,12$; $D P=2,22)$, observando-se predominância do sexo masculino $(53,7 \% ; n=116)$.

Durante o estudo, o questionário OM-6 foi completado pelos cuidadores sem assistência, o que anulou um possível viés resultante da interação com o entrevistador. Todos os cuidadores completaram com sucesso o preenchimento da versão portuguesa do questionário OM-6, tendo o tempo médio de preenchimento do questionário sido de dois minutos.

A avaliação da validade de construção da versão portuguesa do questionário OM-6 revelou coeficientes de correlação de Spearman acima de 0,97 para todos os grupos

Tabela 1 - Resultados da avaliação da 'segunda tradução de consenso' pelo grupo de tradutores

\begin{tabular}{llllllll}
\hline & I & II1 & II2 & II3 & II4 & II5 & II6 \\
\hline 'Percebo muito bem' & $33(66 \%)$ & $28(56 \%)$ & $31(62 \%)$ & $33(66 \%)$ & $32(64 \%)$ & $32(64 \%)$ & $33(66 \%)$ \\
'Percebo bem' & $15(30 \%)$ & $22(44 \%)$ & $17(34 \%)$ & $13(26 \%)$ & $17(34 \%)$ & $16(32 \%)$ & $16(32 \%)$ \\
'Percebo mal' & $2(4 \%)$ & $0(0 \%)$ & $2(4 \%)$ & $3(6 \%)$ & $1(2 \%)$ & $1(2 \%)$ & $1(2 \%)$ \\
'Percebo muito mal' & $0(0 \%)$ & $0(0 \%)$ & $0(0 \%)$ & $1(2 \%)$ & $0(0 \%)$ & $1(2 \%)$ & $0(0 \%)$ \\
\hline
\end{tabular}


Tabela 2 - Avaliação da validade de construção da versão portuguesa do questionário OM-6

\begin{tabular}{|c|c|c|c|c|c|c|}
\hline & \multicolumn{2}{|c|}{ OM-6 } & \multirow[b]{2}{*}{$p$} & \multicolumn{2}{|c|}{ Score de mudança } & \multirow[b]{2}{*}{$p$} \\
\hline & $\mathbf{n}$ & $r_{\mathrm{s}}$ & & $\mathbf{n}$ & $r_{\mathrm{s}}$ & \\
\hline Total da amostra & 216 & $-0,98$ & $<0,001$ & 60 & 0,97 & $<0,001$ \\
\hline \multicolumn{7}{|l|}{ Por sexo } \\
\hline Masculino & 116 & $-0,97$ & $<0,001$ & 29 & 0,93 & $<0,001$ \\
\hline Feminino & 100 & $-0,98$ & $<0,001$ & 31 & 0,99 & $<0,001$ \\
\hline \multicolumn{7}{|l|}{ Por idade } \\
\hline$<5$ anos & 132 & $-0,98$ & $<0,001$ & 42 & 0,96 & $<0,001$ \\
\hline$\geq 5$ anos & 84 & $-0,97$ & $<0,001$ & 18 & 0,99 & $<0,001$ \\
\hline
\end{tabular}

Tabela 3 - Avaliação da consistência interna da versão portuguesa do questionário OM-6

\begin{tabular}{|c|c|c|c|c|}
\hline & $\alpha$ de Cronbach & Correlação inter-item & Correlação item-total & a se item apagado \\
\hline OM-6 & 0,780 & 0,369 & & \\
\hline Sofrimento físico & & & 0,644 & 0,717 \\
\hline Perda auditiva & & & 0,276 & 0,805 \\
\hline Alteração da fala & & & 0,367 & 0,783 \\
\hline Sofrimento emocional & & & 0,663 & 0,715 \\
\hline Limitação de atividade & & & 0,615 & 0,727 \\
\hline Preocupações do cuidador & & & 0,632 & 0,719 \\
\hline
\end{tabular}

$(p<0,001)$, o que traduz uma forte correlação entre a pontuação final obtida na versão portuguesa do questionário OM-6 e os valores obtidos na escala visual analógica de 10 pontos. A correlação foi negativa, dado que a uma pontuação mais elevada no questionário OM-6 corresponde uma pior qualidade de vida relacionada com OM na escala visual analógica de 10 pontos (Tabela 2).

A avaliação da consistência interna da versão portuguesa do questionário OM-6 revelou um valor de alfa Cronbach para todos os domínios em conjunto de 0.780. A análise mostrou uma melhoria ligeira da consistência interna do instrumento com a eliminação dos domínios 'perda auditiva' e 'alterações da fala'. No entanto, a avaliação global da versão portuguesa do questionário OM-6 apresenta um alfa de Cronbach adequado (Tabela 3).

Trinta e quatro cuidadores de crianças com doença estável completaram um segundo questionário no intervalo de sete dias para avaliação da confiança teste-reteste. Esta segunda amostra de conveniência foi composta por 15 crianças do sexo masculino $(44,1 \%)$ e 19 crianças do sexo feminino (55,9\%), com idades compreendidas entre 2 e 12 anos $(M=4,85$; $D P=2,55)$. A normalidade das duas pontuações obtidas (pontuação final inicial e pontuação final reteste) foi avaliada através do teste Kolmogorov-Smirnov. Ambas as variáveis seguiram uma distribuição normal ( $z$ $=0,958 ; p=0,318$ e $z=0,880 ; p=0,421$, respetivamente). Desta forma, utilizámos o coeficiente de correlação de Pearson para avaliação da confiança teste-reteste, tendo sido obtida uma correlação positiva, forte e estatisticamente significativa entre as pontuações finais nos dois momentos de avaliação, no total da amostra e em todos os grupos, com valores de correlação que variaram entre $r=0,82 ; p<$ 0,001 (sexo masculino) e $r=0,89 ; p<0,001$ (total da amos- tra e grupo com $^{3}$ cinco anos) (Tabela 4).

Sessenta cuidadores completaram o questionário dois meses após a colocação de tubos de ventilação transtimpânicos para avaliação da resposta a alteração clínica. Esta terceira amostra de conveniência foi constituída por 29 crianças do sexo masculino $(48,3 \%)$ e 31 do sexo feminino $(51,7 \%)$, com idades compreendidas entre 1 e 12 anos $(\mathrm{M}=3,82 ; \mathrm{DP}=2,11)$. Todas as crianças apresentavam, à data da avaliação de follow-up, tubos de ventilação transtimpânicos in situ e com lúmen patente. Foram encontradas diferenças estatisticamente significativas entre os dois momentos de avaliação com $t(59)=10,104 ; p<0,001$, o que traduz uma melhoria da qualidade de vida das crianças com otite média crónica com efusão e otite média aguda recorrente com o procedimento cirúrgico (Tabela 5). O score de mudança global médio foi de 1,70 (variação: de -0,7 a 4,3 ; DP $=1,30$ ), o que traduz em termos globais uma grande melhoria da qualidade de vida relacionada com o ouvido médio após o procedimento cirúrgico.

A avaliação da validade de construção do score de mudança do questionário OM-6 revelou uma forte correlação

Tabela 4 - Avaliação da confiança teste-reteste da versão portuguesa do questionário OM-6

\begin{tabular}{llll}
\hline & $\mathbf{n}$ & $\boldsymbol{r}_{\mathrm{s}}$ & $\boldsymbol{p}$ \\
\hline $\begin{array}{l}\text { Total da amostra } \\
\text { Por sexo }\end{array}$ & 34 & 0,89 & $<0,001$ \\
$\quad$ & & \\
$\begin{array}{l}\text { Masculino } \\
\text { Feminino }\end{array}$ & 15 & 0,82 & $<0,001$ \\
$\begin{array}{l}\text { Por idade } \\
<5 \text { anos }\end{array}$ & 19 & 0,87 & $<0,001$ \\
$\geq 5$ anos & 20 & & \\
\hline
\end{tabular}


Tabela 5 - Avaliação da resposta a alteração clínica da versão portuguesa do questionário OM-6

\begin{tabular}{|c|c|c|c|c|c|c|}
\hline & \multicolumn{2}{|l|}{ Basal } & \multicolumn{2}{|c|}{ Follow-up } & \multirow{2}{*}{$t$} & \multirow{2}{*}{$p$} \\
\hline & M & DP & M & DP & & \\
\hline Pontuação final & 3,21 & 1,44 & 1,51 & 0.62 & 10,104 & $<0,001$ \\
\hline
\end{tabular}

positiva $\left(r_{\mathrm{s}}=0,97, p<0,001\right)$ entre os scores de mudança do questionário OM-6 e da escala visual analógica de 10 pontos. Os resultados mostram que a scores de mudança superiores do questionário OM-6 correspondem scores de mudança superiores na escala visual analógica de 10 pontos (Tabela 2).

\section{DISCUSSÃO}

A qualidade de vida tem sido reconhecida de forma crescente na investigação clínica, sendo uma importante medida de avaliação de resultados em saúde. O interesse científico pelas medidas de qualidade de vida na população pediátrica, traduzido pelo seu consequente refinamento conceitual e metodológico, visa a melhoria das práticas assistenciais dirigidas a esta população.

A OM é uma das doenças mais comuns na infância, apresentando impacto considerável na qualidade de vida relacionada com a saúde. ${ }^{13-16} \mathrm{~A}$ extensão exata do impacto da OM sobre a qualidade de vida das crianças portuguesas não é conhecida, devido à inexistência de um instrumento em língua portuguesa doença-específico validado para esse efeito. Nesse sentido, considerámos pertinente a criação de uma versão em língua portuguesa e culturalmente adaptada a Portugal do questionário OM- $6,{ }^{3}$ o instrumento mais usado em todo o mundo para avaliação da qualidade de vida relacionada com a saúde em crianças com OM.

A qualidade de vida relacionada com a saúde é um conceito multidimensional. A sua avaliação considera sintomas físicos, capacidade funcional e impacto psicossocial da doença. O questionário OM-6 avalia seis domínios relativos à OM: sofrimento físico, perda auditiva, comprometimento da fala, sofrimento emocional, limitação da atividade e preocupação do cuidador. Possibilita uma avaliação rápida da forma como o cuidador percebe que a criança está a ser afetada pela OM, permitindo a identificação dos domínios com maior importância para a qualidade de vida relacionada com o ouvido médio e o nível de preocupação do cuidador.

O método de tradução adotado para a criação da versão portuguesa do questionário OM-6, com traduções e retro-traduções, considera-se adequado pelo facto de implicar diversas avaliações independentes. ${ }^{17}$ Contudo, deve ser considerada como possível limitação do processo de adaptação cultural do instrumento, já que o mesmo foi aplicado a uma amostra de conveniência de apenas um centro hospitalar. Estudos futuros devem considerar este aspeto, de modo a assegurar uma validade óptima de conteúdo do questionário, quando aplicado em diferentes áreas geográficas.

A versão portuguesa do questionário OM-6 mostrou ser um método válido para avaliação da qualidade de vida re- lacionada com a saúde em crianças com otite média crónica com efusão ou otite média aguda recorrente, uma vez que a pontuação obtida no questionário revelou forte correlação com a pontuação obtida na escala visual analógica (validade de construção). A escala visual analógica é uma medida externa adequada para avaliação da validade de construção da versão portuguesa do questionário OM-6, dado ser um instrumento válido, confiável e sensível, comparável aos questionários com múltiplos itens de avaliação, estando recomendada a sua utilização para avaliação global da qualidade de vida em ensaios clínicos. ${ }^{18} \mathrm{~A}$ escala visual analógica mostrou em estudos anteriores ser uma ferramenta adequada para avaliação global da qualidade de vida de crianças com OM antes e após o tratamento, podendo ser usada como um método simplificado de análise da eficácia terapêutica neste grupo de doentes. ${ }^{19}$

A versão portuguesa do questionário OM-6 apresentou correlação entre os resultados de itens semelhantes (consistência interna). Contudo, a análise mostrou uma meIhoria ligeira da consistência interna do instrumento com a eliminação dos domínios 'perda auditiva' e 'alterações da fala'. Este aspeto pode ser justificado pela idade das crianças, dado que muitas se encontravam em estádios mais precoces do desenvolvimento da fala $(61,1 \%)$, o que pode resultar em dificuldade por parte do cuidador em avaliar corretamente alterações da fala. Por outro lado, a capacidade do cuidador para avaliar a presença de perda auditiva é igualmente questionável. A perda auditiva é um sintoma importante da otite média com efusão e um dos domínios avaliados pelo questionário OM-6. No entanto, estudos que aplicaram este questionário mostraram uma fraca correlação entre a perceção da audição pelos cuidadores e os resultados dos exames audiológicos. ${ }^{3,8,20}$ Também a perceção da alteração da acuidade auditiva não mostrou correlação com a alteração audiológica efectiva. ${ }^{3}$ Deste modo, embora a perceção da audição pelos cuidadores seja de extrema importância na determinação da qualidade de vida das crianças com OM, a mesma deve ser sempre complementada por avaliação audiológica. Apesar da possível limitação dos domínios 'perda auditiva' e 'alterações da fala', optámos por não eliminar nenhum dos domínio de avaliação da versão portuguesa do questionário OM-6, dado que ambos cobrem preocupações fundamentais na $\mathrm{OM}$, não sendo a perda de conteúdo compensada por nenhum dos outros domínios, e a avaliação global do instrumento apresentar um $\alpha$ - Cronbach adequado.

A versão portuguesa do questionário OM-6 mostrou igualmente produzir resultados relativamente estáveis quando readministrado em circunstâncias de doença estável (confiança teste-reteste) no total da amostra e em todos os grupos, sendo a confiabilidade teste-reteste altamente 
dependente da estabilidade clínica da amostra em estudo.

As crianças submetidas a colocação de tubos de ventilação transtimpânicos como parte do seu seguimento clínico apresentaram uma melhoria significativa da qualidade de vida relacionada com o ouvido médio. A versão portuguesa do questionário OM-6 mostrou, deste modo, capacidade de quantificar mudanças da qualidade de vida clinicamente significativas ao longo do tempo num mesmo doente (resposta a alteração clínica). Os resultados apresentados não procuram suportar a eficácia dos tubos de ventilação transtimpânicos, mas demonstrar a utilidade clínica do questionário OM-6 em disponibilizar, através do score de mudança, uma medida facilmente interpretável da direção da alteração clínica (melhoria/agravamento) e da sua magnitude (<0,5 trivial; 0,5 - 0,9 pequena; $1,0-1,4$ moderada; $\geq 1,5$ grande).

Os instrumentos desenvolvidos mais recentemente para avaliação da qualidade de vida na população pediátrica valorizam de forma crescente o ponto de vista da criança, envolvendo-a mais diretamente nas decisões sobre si mesma. Contudo, a construção de instrumentos de avaliação da qualidade de vida em pediatria apresenta algumas limitações. A auto-avaliação da criança sobre a sua qualidade de vida é influenciada pela capacidade de compreensão verbal, ou seja, a criança deve entender o que lhe está a ser perguntado para depois ser capaz de formular a resposta. Por outro lado, muitas das medidas de qualidade de vida requerem que a resposta seja baseada nas experiências durante um período específico de tempo, como se verifica no questionário OM-6, sendo que a capacidade da criança responder com relação ao tempo é influenciada pelo desenvolvimento da memória e da perceção temporal. ${ }^{21}$ Deste modo, na maioria dos casos, a única forma de obter informação sobre a qualidade de vida é recorrer a fontes alternativas, como os cuidadores, pedindo que reflitam sobre a perceção da qualidade de vida da criança, o que pode não ser necessariamente concordante. ${ }^{22}$ No entanto, estudos anteriores concluíram que a avaliação da qualidade de vida pelo cuidador correlaciona-se de forma apropriada com a auto-avaliação da criança relativamente ao seu sofrimento físico e a outros sintomas observáveis, o que sugere que cuidadores próximos são aceitáveis para responder a questionários que avaliem esses domínios, como é o caso do questionário OM-6.

Deve também considerar-se que os indicadores de qualidade de vida variam de acordo com a idade. ${ }^{21}$ Cada item do questionário OM-6 está estruturado como um domínio completo, dentro do qual o cuidador pode realizar a sua graduação de impacto de sintomas de acordo com a idade, o que permite contornar as limitações decorrentes da avaliação nas diferentes fases de desenvolvimento.

Estudos indicam que os cuidadores sobrestimam a qualidade de vida relacionada com a saúde em crianças com OM antes do tratamento (response shift). Após a abordagem terapêutica, os cuidadores percebem que a qualidade de vida antes do tratamento era inferior ao que tinham reconhecido, através da comparação do estado clínico da crian- ça antes e após a cirurgia (scale recalibration). Os resultados sugerem que a natureza dinâmica dos instrumentos de avaliação da qualidade de vida deve ser considerada, sob o risco de haver uma subestimação do efeito terapêutico. ${ }^{8}$

É expectável a possibilidade de observar-se uma dissociação entre o estado do ouvido médio e a avaliação da qualidade de vida. A otite média crónica com efusão caracteriza-se frequentemente por uma escassez de sintomas, podendo acompanhar-se de uma pontuação inferior no questionário embora a criança apresente alterações óbvias do ouvido médio. Por outro lado, crianças com um ouvido médio normal podem apresentar pontuações superiores no contexto de otite média aguda recorrente dado que, embora transitória, cursa com afeção significativa da qualidade de vida. Deste modo, medidas objetivas do estado do ouvido médio e medidas subjetivas de qualidade de vida devem ser consideradas simultaneamente na avaliação do estado clínico das crianças com OM, no estabelecimento de expectativas relativas ao tratamento e na determinação do sucesso terapêutico.

O questionário OM-6 não é um instrumento discriminativo, uma vez que a pontuação final e dos domínios individuais não é válida para comparação entre indivíduos. Embora cada domínio avalie um conjunto de sintomas e a resposta dos cuidadores de diferentes crianças dificilmente seja baseada nos mesmos sintomas, respostas repetidas de um mesmo cuidador de uma criança específica podem ser comparadas, representando uma medida válida para avaliação da resposta clínica dessa criança.

O questionário OM-6 permite obter dados objetivos sobre a qualidade de vida relacionada com o ouvido médio. A simplicidade e brevidade de aplicação do instrumento tornam-no ideal para avaliação da qualidade de vida na prática clínica diária e não apenas em ensaios clínicos e estudos observacionais.

\section{CONCLUSÃO}

A versão portuguesa do questionário OM-6 é um instrumento válido, confiável e sensível para avaliação da qualidade de vida relacionada com saúde em crianças com OM. Acreditamos que a sua utilização na prática clínica e na investigação permitirá a caracterização do impacto que esta patologia apresenta na qualidade de vida das crianças portuguesas e a avaliação do sucesso das medidas terapêuticas, o que se traduzirá numa consequente melhoria dos cuidados de saúde prestados.

\section{OBSERVAÇÕES}

Parte do trabalho foi apresentado em formato de comunicação livre, no $63^{\circ}$ Congresso da Sociedade Portuguesa de Otorrinolaringologia e Cirurgia Cérvico-Facial, no dia 24 de Abril de 2016, em Coimbra.

\section{PROTECÇÃO DE PESSOAS E ANIMAIS}

Os autores declaram que os procedimentos seguidos estavam de acordo com os regulamentos estabelecidos pelos responsáveis da Comissão de Investigação Clínica 
e Ética e de acordo com a Declaração de Helsínquia da Associação Médica Mundial.

\section{CONFIDENCIALIDADE DOS DADOS}

Os autores declaram ter seguido os protocolos do seu centro de trabalho acerca da publicação de dados.

\section{CONFLITOS DE INTERESSE}

Os autores declaram não terem qualquer conflito de interesse relativamente ao presente artigo.

\section{FONTES DE FINANCIAMENTO}

Estudo realizado sem recurso a subsídio ou bolsa.

\section{REFERÊNCIAS}

1. Speets A, Wolleswinkel J, Cardoso C. Societal costs and burden of otitis media in Portugal. J Multidiscip Healthc. 2011;4:53-62.

2. Paparella M, Schachern P, Yoon T, Abdelhammid M, Sahni R, Da Costa S. Otopathologic correlates of the continuum of otitis media. Ann Otol Rhinol Laryngol. 1990;148:S17-22

3. Rosenfeld RM, Goldsmith AJ, Tetlus L, Balzano A. Quality of life for children with otitis media. Arch Otolaryngol Head Neck Surg. 1997; 123:1049-54.

4. Heikkinen T, Ruuskanen O. Signs and symptoms predicting acute otitis media. Arch Pediatr Adolesc Med. 1995;149:26-9.

5. Kontiokari T, Koivunen P, Niemelä M, Pokka T, Uhari M. Symptoms of acute otitis media. Pediatr Infect Dis J. 1998;17:676-9.

6. Paradise JL. Otitis media and child development: should we worry? Pediatr Infect Dis J. 1998;17:1076-83.

7. Paradise JL, Dollaghan CA, Campbell TF, Feldman HM, Bernard BS, Colborn DK, et al. Language, speech sound production, and cognition in three-year-old children in relation to otitis media in their first three years of life. Pediatrics. 2000;105:1119-30.

8. Timmerman AA, Anteunis LJ, Meesters CM. Response-shift bias and parent-reported quality of life in children with otitis media. Arch Otolaryngol Head Neck Surg. 2003;129:987-91.

9. Heidemann $\mathrm{CH}$, Godballe $\mathrm{C}$, Kjeldsen $\mathrm{AD}$, Johansen $\mathrm{EC}$, Faber $\mathrm{CE}$, Lauridsen $\mathrm{HH}$. The Otitis Media-6 questionnaire: psychometric properties with emphasis on factor structure and interpretability. Health Qual Life Outcomes. 2013;11:201.

10. Li G, Qiyun G, Guohua D, Tingting C, Dan L. Assessment on the quality of life for children with secretory otitis media after tympanostomy tube combined adenoidectomy. Chin J Otol. 2016;14:396-9.

11. Gebhart DE. Tympanostomy tubes in otitis prone child. Laryngoscope. 1981;91:849-66.

12. Mandel EM, Rockette HE, Bluestone CD, Paradise JL, Nozza RJ. Efficacy of myringotomy with and without tympanostomy tubes for

chronic otitis media with effusion. Pediatr Infect Dis J. 1992;11:270-7.

13. Brouwer CN, Maillé AR, Rovers MM, Grobbee DE, Sanders EA, Schilder AG. Health-related quality of life in children with otitis media. Int J Pediatr Otorhinolaryngol. 2005;69:1031-41.

14. Grindler DJ, Blank SJ, Schulz KA, Witsell DL, Lieu JE. Impact of otitis media severity on children's quality of life. Otolaryngol Head Neck Surg. 2014;151:333-40.

15. Blank SJ, Grindler DJ, Schulz KA, Witsell DL, Lieu JE. Caregiver quality of life is related to severity of otitis media in children. Otolaryngol Head Neck Surg. 2014;151:348-53.

16. Lee J, Witsell DL, Dolor RJ, Stinnett S, Hannley M. Quality of life of patients with otitis media and caregivers: a multicenter study. Laryngoscope. 2006;116:1798-804.

17. Beaton DE, Bombardier C, Guillemin F, Ferraz MB. Guidelines for the process of cross-cultural adaptation of self-report measures. Spine. 2000;25:3186-91.

18. de Boer AG, van Lanschot JJ, Stalmeier PF, van Sandick JW, Hulscher $\mathrm{JB}$, de Haes JC, et al. Is a single-item visual analogue scale as valid, reliable and responsive as multi-item scales in measuring quality of life? Qual Life Res. 2004;13:311-20.

19. Datema FR, Vemer-van den Hoek JG, Wieringa MH, Mulder PM, Baatenburg de Jong RJ, Blom HM. A visual analog scale can assess the effect of surgical treatment in children with chronic otitis media with effusion. Int J Pediatr Otorhinolaryngol. 2008;72:461-7.

20. Escada P. A capacidade de previsão, por parte dos familiares, da acuidade auditiva das crianças com otite média. Lisboa: Faculdade de Ciências Médicas da Universidade Nova de Lisboa; 2002.

21. Wallander JL, Schmitt M, Koot MH. Quality of life measurement in children and adolescents: issues, instruments and applications. J Clin Psychol. 2001;57:571-85.

22. Eiser C, Morse R. Can parents rate their child's health-related quality of life? Results of a systematic review. Qual Life Res. 2001;10:347-57. 